\title{
Principles and Fundamentals of Optical Imaging
}

\section{Frank Scheffold}

\begin{abstract}
In this chapter I will give a brief general introduction to optical imaging and then discuss in more detail some of the methods specifically used for imaging cortical dynamics today. Absorption and fluorescence microscopy can be used to form direct, diffraction-limited images but standard methods are often only applicable to superficial layers of cortical tissue. Two-photon microscopy takes an intermediate role since the illumination pathway is diffraction-limited but the detection pathway is not. Losses in the illumination path can be compensated using higher laser power. Since the detection pathway does not require image formation, the method can substantially increase the imaging depth. Understanding the role of scattering is important in this case since non-descanned detection can substantially enhance the imaging performance. Finally, I will discuss some of the most widely used imaging methods that all rely on diffuse scattering such as diffuse optical tomography, laser speckle imaging, and intrinsic optical imaging. These purely scattering-based methods offer a much higher imaging depth, although at a substantially reduced spatial resolution.
\end{abstract}

Key words Optical imaging, Fluorescence microscopy, Scattering, Diffuse optical tomography, Laser Speckle Imaging

\section{Introduction}

Modern optical imaging relies on a number of optical phenomena that provide access to spatial and temporal information about the object under study. Sample-specific information can be extracted not only from the intensity or phase but also from the analysis of photon statistics, fluorescence lifetime, polarization, and so on [1]. Here I will give a brief general introduction to optical imaging and then discuss in more detail some of the methods specifically used for imaging dynamics of cerebral cortex today. Imaging cortical dynamics often mandates the use of specific indicators, requires deep tissue penetration or high temporal resolution. Methods prominently discussed in this volume are two-photon microscopy, absorption spectroscopy, and laser speckle imaging. Although these methods are based on different principles, they all are intimately related to (multiple) light scattering and absorption, topics 
that often are not treated in classical optical imaging books and reviews and therefore are highlighted here.

I will first discuss the main mechanism of light-matter interactions in biological tissue, namely scattering, absorption, and fluorescent light emission. In the next step, I address the fundamentals of image formation for some selected methods and discuss their advantages and limitations.

\section{Imaging and Microscopy Principles}

An image in traditional light microscopy is formed by absorption or scattering of light waves. In essence the microscope records the transmission shadow of a section of the sample that is in focus. The depth of field $Z$ denotes the thickness of a $\mathrm{z}$-section around the focal plane for which object points are imaged with acceptable sharpness. Light beams scattered or absorbed at a given lateral $(x-y)$ position, within this section $Z$, are removed and therefore this $x-y$ point appears dark in the image plane. Light beams unaffected by the sample lead to a bright background. The difference between dark and bright areas, or low and high light amplitudes, is called "contrast."

Absorption is a molecular process where the photon energy is converted via radiative and non-radiative pathways in the molecule (see below). Refractive index variations $n(x, y, z)$ in the sample can lead to scattering or phase changes or both. Phase contrast originates from differences in the optical light path, defined as $s=x c$. Here $x$ is the geometrical path, $c=c_{0} / n$ the speed of light and $c_{0}$ denotes the vacuum speed of light. Roughly speaking one can say that refractive index variations on length scales comparable to the wavelength $\lambda$, here approximately $500 \mathrm{~nm}$, lead to scattering while variations on much larger length scales mainly lead to phase shifts. Phase shifts can be converted to amplitude changes using the socalled phase plates in phase contrast microscopy. Applications are mainly found for unstained biological sections that are weakly scattering objects. For details, please refer to the standard textbooks.

For all these cases, image formation can be treated with Abbe's classical coherence theory for image formation: The lateral resolution is set by the effective numerical aperture (NA) of the objective and the condenser to $\rho_{\min }=0.61 \lambda / \mathrm{NA}$ and the depth of field is $Z=n \lambda / \mathrm{NA}^{2}$ [2]. In addition to the lateral resolution limit set by the diffraction of light the utility of classical microscopy for biomedical imaging is severely limited by other factors. In particular the lack of specificity, the limited axial resolution and the poor penetration depth in optically dense media have led to the development of a large number of new methods based on other principles of contrast formation [1].

Fluorescence microscopy is nowadays the method of choice in many imaging applications. The interaction of light with fluorescent 


\subsection{Absorption and Fluorescence}

molecules offers several advantages. Firstly the molecules can be attached to specific sites and act as specific indicators for biological function. Since each molecule emits light at wavelengths within a certain spectral window, it is possible to separate several different fluorescent markers in a single experiment. As a matter of fact, biological specimen often display limited intrinsic amplitude or phase contrast, thus fluorescence microscopy often provide images of unrivalled quality.

Although a source of contrast for thin samples, absorption and scattering usually impose severe limitations on the penetration depth when imaging thick specimen. If the absorbed light is not reemitted (e.g., via fluorescence), the energy relaxes via vibrational pathways and the photons are lost. In the case of scattering however, the light is merely redirected. Although this precludes classic image formation in a microscope, it still allows to reconstruct lower resolution images based on diffuse propagation of scattered photons [3]. Collection of scattered photons is also an important issue in the detection pathway of two-photon microscopy [4]. In cases where the scattering has an inelastic or Doppler-contribution due to microscopic internal motion, additional information is encoded in the propagating light fields that carry information, for example, about blood flow in tissue [5]. In the following I describe in more detail the main principles of image formation for a number of cases that are of importance for cortical imaging.

Light energy can be "absorbed" by matter and subsequently released via radiative and non-radiative pathways. The latter process transforms the entire energy of an incident photon to thermal energy. Absorption without emission can provide amplitude contrast in image formation but it can also severely limit the penetration depth of optical imaging methods. Radiative decays result in the emission of photons via fluorescence or phosphorescence. In complex molecules both radiative and non-radiative processes occur sequentially or concurrently. As a consequence, for single photon absorption, the emitted photon possesses a lower energy (or has a red-shifted wavelength) compared to the absorbed photon. The situation can be described using the so-called Jablonski diagrams depicting the energy states of a molecule and its relaxation pathways (Fig. 1) [6].

In contrast to a single atom, the electronic states of a complex molecule have many associated vibrational energy levels. In practice a molecule is excited from one of these thermally excited vibrational levels of a singlet ground state $S_{0}$ to an exited single state $S_{1}$ or $S_{2}$ by absorbing a photon. The molecular absorption spectrum is rather broad, typically tens of nanometers. The excited molecule can relax to the ground state via different pathways. Non-radiative transitions are described via dashed lines whereas solid line arrows describe radiative pathways (Fig. 1). "Fluorescence" is the process 


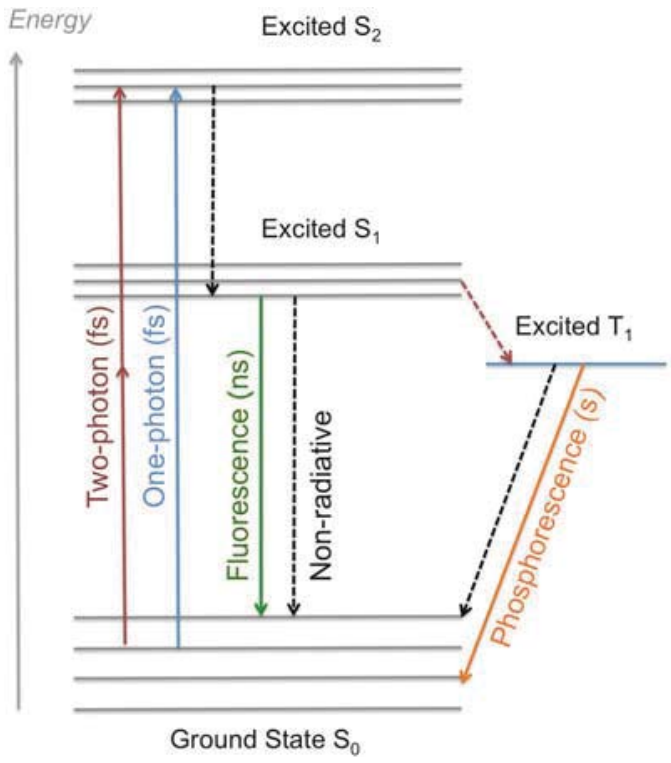

Fig. 1 Jablonski diagram. Schematic representation of the energy levels of an electron in a fluorescent molecule (typical relaxation time scales in brackets). The molecule can be excited by $1 p$ or $2 p$ absorption to a higher electronic state $S_{2}$ or $S_{1}$. Several radiative and non-radiative pathways eventually lead to a collapse to the ground state $S_{0}$. The term "fluorescence emission" denotes the fast radiative decay from the lowest vibrational level of $S_{1}$ to an excited ground state $S_{0}$ level (green arrow).

of fast emission of a photon via a direct relaxation from a low energy vibrational state of $S_{1}$ to $S_{0}$, which typically takes place on the nanosecond scale. The exact lifetime of the excited state of a fluorescent molecule depends on its immediate environment and thus carries relevant morphological and often also functional information. An image of these properties is constructed by pointscanning in the frame the fluorescence lifetime imaging microscopy (FLIM) approach [7]. If the molecule first undergoes a spin conversion, it can end up in a "forbidden" triplet state $T_{1}$. A subsequent radiative decay is slow and the photon energy is emitted over time scales of a millisecond or more, a process known as "phosphorescence." For imaging purposes, mostly fluorescence is employed whereas the other processes merely lead to a reduced quantum efficiency and increased background. In some cases, however, these other processes can carry specific information. Fluorescence can be suppressed by resonant energy transfer (FRET), provided the molecules are sufficiently close to each other [8]. The efficiency of this process scales with the sixth power of the distance and the signal (or the absence of the signal) thus carries information about the location of the molecules at the nanoscale. Equally phosphorescence can be quenched, for example, by the presence of oxygen $[6,9,10]$. 


\subsection{Scattering and} Multiple Scattering
Standard fluorescence microscopy is based on the absorption of one high energy photon, some non-radiative losses and the subsequent emission of a lower energy photon: for example, blue excitation light is turned into green emission light (Fig. 1). In contrast, in two-photon microscopy a pair of photons is absorbed simultaneously and the fluorescent molecule emits a single higher energy photon: for example, near infrared (NIR) light is turned into green light (Fig. 1). In the latter case the fluorophores normally do not absorb single low energy NIR-photons and thus twophoton excited fluorescence is the only allowed process.

The scattering of light refers to the elastic or quasi-elastic interaction of light with matter where the exchange of energy is minimal. In the latter case, for all cases of practical interest, the frequency shift $\Delta \nu$ due to scattering from moving objects is very small (much smaller than the line-width of a single frequency laser of typically $5-10 \mathrm{MHz}$ ), which means that the scattering process itself does not alter the properties of the scattered light. Scattering occurs due to variations in the refractive index of the medium on length scales of the wavelength of light. The incident wave induces oscillating dipoles in the medium that can reemit part of the light energy in some direction. Both scattering and absorption perturb the light propagation such that for a given path of light $z$ the stream of photons is attenuated according to Lambert-Beers law $I(z)=I_{0} \exp (-A)=I_{0} \exp (-\mu z)$. The scattering and absorption strengths are characterized by the parameters $\mu_{\mathrm{s}}$ and $\mu_{\mathrm{a}}$, respectively (see Box 1 ).

\section{Box 1 Scattering Efficiency}

The efficiency of scattering is directly related to the polarizability $\alpha$ of the scattering object [11]. In the weak scattering limit $\left[4 \pi R\left(n / n_{0}-1\right) \ll \lambda\right], \alpha$ is proportional to the refractive index contrast $\left(n / n_{0}-1\right)$. Here $n$ denotes the refractive index of the object and $n_{0}$ of the background medium and $\lambda$ is the wavelength of light. For small objects of size $R$, one finds $\alpha \propto R^{3}$ $(R<\lambda / 2 \pi)$ and for larger particles $\alpha \propto R^{2}(R>\lambda / 2 \pi)$. The total scattering cross section is proportional to $\alpha^{2}$. Both the influence of absorption and scattering are usually measured in terms of the parameters $\mu_{\mathrm{a}}$ and $\mu_{\mathrm{s}}$ with the latter being directly proportional to $\alpha^{2}$ and the number density of scatterers. The parameter $\mu_{\mathrm{s}}=1 / l_{\mathrm{s}}$ is equal to the inverse of the mean free path $l_{\mathrm{s}}$ defined as the distance a wave can travel on average before being scattered. In other words the unscattered or "ballistic" light intensity decreases exponentially $I=I_{0} \exp \left[-z / l_{\mathrm{s}}\right]$ as a function of the penetration depth $z$ (LambertBeer law). Objects of size comparable or larger than the wavelength of light, such as cells and other tissue structures, scatter preferentially in forward direction. A measure of this effect is the scattering anisotropy parameter defined as the average of the cosine of the scattering angle $g=\langle\cos \Theta\rangle$. For objects much smaller than the wavelength $g \simeq 0$ whereas for biological tissue and blood, it is typically of the order $g=0.9$. Diffuse propagation of light is characterized by the parameter $\mu_{\mathrm{t}}=1 / h_{\mathrm{t}}$ that takes account of the scattering anisotropy via a renormalized path length $l_{\mathrm{t}}=l_{\mathrm{s}} 1 /(1-g)$. 




Fig. 2 Optical properties of blood. Scattering coefficient $\mu_{\mathrm{s}}$ and absorption coefficient $\mu_{\mathrm{a}}$ of oxygenated (red) and deoxygenated (blue) whole blood, both adapted from Faber et al. [13].

Scattering in biological tissue is either due to refractive index variations in the tissue or due to scattering from blood cells. Scattering from blood can play an important role, for example, for in vivo two-photon imaging, given the high vascularization of the brain cortex [12]. Figure 2 shows the scattering properties of whole blood for light in the visible (VIS) and NIR region. For blue and green light, absorption and scattering are of similar strength while in the red and NIR region absorption decreases to a level where it becomes negligible for most applications. Scattering, however, remains strong in this wavelength range and limits the penetration depth of light in many optical imaging methods. As can be seen from Fig. 2 the scattering coefficient $\mu_{\mathrm{s}}$ for whole blood never drops below $200 \mathrm{~mm}^{-1}$ and thus mean free path $l_{\mathrm{s}}$ never exceeds a value of $5 \mu \mathrm{m}$. Therefore whole blood is almost impenetrable for microscopy methods. The blood content in tissue varies substantially but is typically of the order of $2-5 \%$. At the same time refractive index variations in (cortical) tissue also provide scattering contrast. As a consequence, regular tissue, such as brain grey matter, for example, has a scattering mean free path of about $100-200 \mu \mathrm{m}$ in vivo and negligible absorption in the $\operatorname{NIR}[4,14]$ (see also Chapter 3 in this volume).

\section{Single-Photon Microscopy}

There are two distinct ways of obtaining an image from a fluorescently labeled specimen upon single-photon excitation: (a) wide field detection and (b) laser scanning combined with confocal detection. Wide-field fluorescence microscopy essentially works the same way as standard microscopy. The only difference is the 
wavelength-selective illumination and detection pathways. The main advantages are reduced background, increased contrast, and the possibility to specifically label (multiple) sites of interest in the specimen. The sectioning capabilities are, however, severely compromised by the contribution of out-of-focus fluorescence excitation if the entire sample is illuminated. The latter also leads to blurring of the image and increased background. Numerical reallocation of out-of-focus light by recording a series of focal planes is known as "deconvolution." It allows 3D reconstruction and can improve the focal plane image sharpness [1]. Selective Plane Illumination Microscopy (SPIM) is an interesting new widefield imaging approach with improved sectioning capabilities. The method is based on creating a sheet of light, which coincides with the focal plane of the objective. With this approach, fluorescence is excited only on the plane of measurement, and by moving the object through this excitation plane it is possible to recover $3 \mathrm{D}$ information with microscopic resolution $[15,16]$.

The most popular approach to achieve 3D high-resolution microscopy is confocal laser scanning microscopy. In CLSM out-of-focus light is reduced by physical mechanism: First, the sample is illuminated with a focused laser beam that is scanned across the specimen. Second, out-of-focus emitted light is rejected by placing a small aperture (the "pinhole") in the image plane of the focal spot. The light that passes the pinhole is then recorded using an electronic detector. A 2D or 3D image is obtained by scanning the sample or the beam [2]. Confocal microscopy allows $3 \mathrm{D}$ microscopy at the Abbe resolution limit for multicolor-labeled samples and is thus widely used in biomedical imaging. The penetration depth in tissue is however limited in scattering samples since a sharp image of the focal plane must be formed on the detection aperture (Fig. 3).

\section{Two-Photon Microscopy}

Two-photon laser scanning microscopy also uses a raster-scanning approach and an objective lens to focus the incident light $[4,12$, 17, 18]. Firstly it is necessary to provide a high enough light intensity to achieve a decent transition probability for two-photon absorption. In practice this can only be achieved using pulsed lasers with pulse durations in the sub-picosecond range and an average power ranging from tens of $\mathrm{mW}$ to several hundreds of $\mathrm{mW}$ for in vivo applications. Provided two-photon excitation can be achieved, the method offers some particular advantages. The two-photon absorption probability scales with the light-intensity square and thus the region where absorption takes place is strongly confined to the focal spot of the focused laser beam. Both axially and laterally the probability decays sharply. All fluorescent light emitted can 

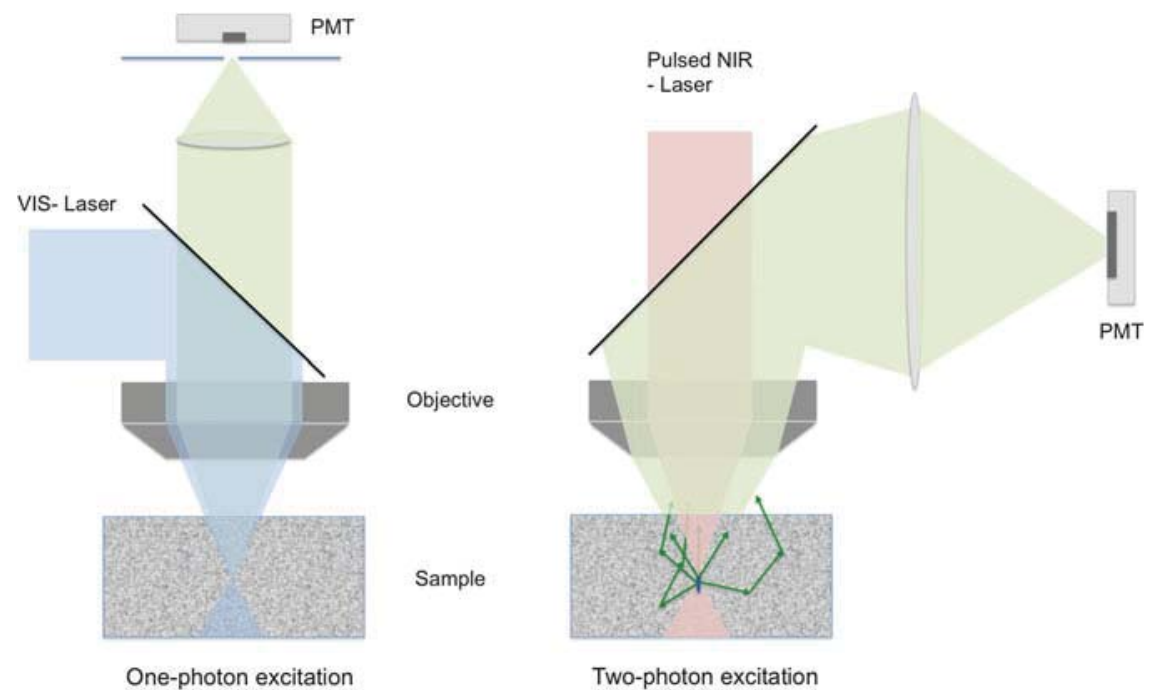

Fig. 3 Laser scanning optical microscopy based on one-photon (left) and two-photon (right) excitation. In both cases scattering reduces the incident power reaching the focal spot. While in one-photon microscopy this leads to background fluorescence, in two-photon microscopy scattered incident light is invisible. In one-photon microscopy (CLSM) illumination and detection pathways are equivalent. Scattered light cannot be imaged sharply on the confocal aperture and is thus lost when forming an image. In two-photon microscopy all emitted photons can be used to construct the image.

therefore be attributed to absorption at the position of the illumination focus. Rejection of out-of-focus light is not required and even scattered light can be collected when constructing an image (Fig. 3). Overall the setup is largely simplified since for the detection pathway a maximum amount of the emitted light is collected. This approach is often called non-descanned detection since, in contrast to confocal microscopy, the reverse pathway does not require the formation of an image of the focal spot. Another important advantage, in particular for biomedical imaging, is the fact that the incident light can be from the NIR region of the spectrum while the emitted light is typically in the VIS range. Both NIR illumination and non-descanned detection strongly increase imaging depth of two-photon microscopy compared to confocal microscopy [4]. Figure 3 summarizes the detection pathways of single- and two-photon excited fluorescence microscopy.

\section{$5 \quad$ Diffuse Light Imaging}

Imaging using microscopy requires a well-defined optical path from illumination to detection. If scattering or absorption is strong, this imposes severe limitations. For in vivo microscopy on small animals or humans, access is commonly restricted to the reflection 
measurements, limiting the optical penetration depth to $0.5-1 \mathrm{~mm}$ even for best case of two-photon microscopy.

It is however still possible to obtain low-resolution images by monitoring scattered light only. The propagation of light in the multiple scattering regime can be described as a random walk of photons with a step length of $l_{\mathrm{t}}=l_{\mathrm{s}} \mathrm{l} /(\mathrm{l}-\mathrm{g})$. For sufficiently large number of steps, light propagation is diffusive with a diffusion coefficient $D=c h_{h} / 3$ [19]. Diffusely reflected light penetrates the sample up to a depth of a few $l_{\mathrm{t}}$ and when leaving the sample spreads out over an area of roughly $10\left(l_{\mathrm{t}}\right)^{2}[20,21]$. In turn, light detected at a given point $(x, y)$ on the surface might originate from any position within the same surface area around this point. The length $l_{t}$ therefore sets a lower bound for the spatial resolution of diffuse reflectance imaging techniques such as optical intrinsic or laser speckle imaging. Imaging contrast can be generated by absorption, fluorescence, and local motion in the sample (Box 2). In the presence of heterogeneities on length scales comparable or larger than $1_{t}$, knowledge about the effective path length is needed as discussed in more detail below. Such knowledge is however often difficult to obtain experimentally. For small animals, this limitation can be overcome using tomography techniques (both for absorption [22] and fluorescence [23]). The situation is much more involved for larger animals and humans when only reflection measurements can be performed. The mathematical description of diffuse light propagation is fairly complicated. A brief primer is given in Box 2.

Box 2 Diffuse Light Propagation: Scattering, Absorption of Light, and Correlation

Contrast in diffuse light imaging can be generated by spatial heterogeneities with respect to scattering $\left(\mu_{\mathrm{t}}\right)$, absorption $\left(\mu_{\mathrm{a}}>0\right)$, and internal motion $f(\tau)$. All four phenomena can be treated within the same light diffusion formalism $[3,24,25]$ and the position-dependent sample properties are characterized by the expression $3 \mu_{\mathrm{t}} \mu_{\mathrm{a}}+f(\tau)$. Stimulated emission can be treated as negative absorption $\left(\mu_{\mathrm{a}}<0\right)$ while fluorescence can also be included in a slightly expanded approach by taking account for the difference in the absorption and emission wavelengths. In the presence of locally increased absorption or fluorescence the mean intensity of light transmitted or reflected from the sample displays spatial variations that can be detected. The function $f(\tau)$ is a measure of the time-dependent microscopic motion, with $f(0)=0$ [26]. Formally it can be considered as an absorption coefficient for the "correlation" of optical fields. Motion gradually destroys the perfect phase correlation of multiply scattered optical fields. For coherent laser light, in the presence of internal motion, light fields fluctuate and $f(\tau)$ increases as a function of time $\tau$. The associated loss of correlation can be probed by studying the optical speckle pattern $[27,28]$. 
Diffuse light imaging can be implemented in a number of different ways as will be described in the following sections $[3,29]$.

\section{Optical Imaging of Intrinsic Signals}

Optical imaging of intrinsic signals (OIS) determines the absorption of reflected light, such as the exposed brain cortex, for one or more often several characteristic wavelengths $([30,31])$. Due to its simplicity, it has been widely applied in an attempt to determine the cortical concentration of oxy- and deoxy-hemoglobin (oxy- $\mathrm{Hb}$, deoxy- $\mathrm{Hb}$ ) during cortical activation $[32,33]$. Using light in the NIR range, optical imaging can also be applied in humans to noninvasively monitor hemoglobin oxygenation $[33,34]$.

A major complication of the OIS technique is the fact that the interpretation of recorded data is not straightforward at all. Since the light propagation in VIS and NIR spectral regions is dominated by the process of multiple scattering, the actual path length within the absorbing tissue, required for quantification, cannot be easily found (see also Box 3). The situation is illustrated in Figs. 2 and 4 . Figure 2 displays the blood absorption and scattering properties for the relevant spectral region. Figure 4 illustrates that the light path can be substantially longer than the penetration depth $z$, which means that the meandering photons have a higher probability of getting absorbed. The situation is further complicated by the fact that the relevant distance travelled within a region of activity (absorption changes or motion) might be much smaller than the total path length [37].

The main problem with the differential path length approach is that experimental access [38] and modeling of $\mathrm{DP}(\lambda)$ in reflection from a heterogeneous medium is very complicated and to date the interpretation of intrinsic optical imaging data remains controversial.

\section{Box 3 The Differential Path Length}

Quantification in optical intrinsic imaging (OIS) relies on the concept of the so-called wavelength-dependent differential path length $\operatorname{DP}(\lambda)$. It is defined such that for the case of absorption the attenuation coefficient is $A(\lambda)=\mathrm{DP}(\lambda) \mu_{\mathrm{a}}$ with $A(\lambda)=-\ln \left[I / I_{0}\right]$. In other words, $\mathrm{DP}(\lambda)$ is the mean path length travelled in a corresponding homogeneous medium with an absorption coefficient $\mu_{\mathrm{a}}$ (Lambert-Beer law). In an actual experiment, it is almost impossible to measure absolute values of $A(\lambda)$ and therefore the analysis is usually restricted to relative changes of the attenuation coefficient: $\partial A(\lambda) / \partial \mu_{\mathrm{a}}=\mathrm{DP}(\lambda)$ or $\Delta \mu_{\mathrm{a}}=\Delta A(\lambda) \mathrm{DP}(\lambda)^{-1}$, where only small changes of $\mu_{\mathrm{a}}$ are considered such that $\operatorname{DP}(\lambda)$ remains unchanged. The knowledge of $\operatorname{DP}(\lambda)$ therefore would allow to relate absorption changes to changes in concentration of the absorbing substance since $\Delta \mu_{\mathrm{a}} \propto$ concentration. 


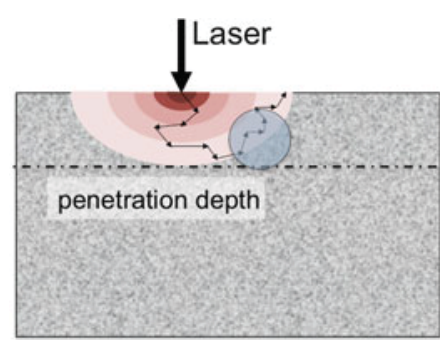

cross section

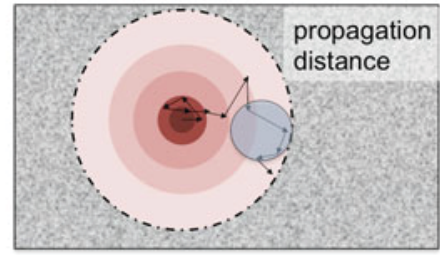

top view

Fig. 4 Diffuse scattering of light in reflection. The incident wave is dispersed and scattered along a path of a certain contour length. The scattered wave leaves the sample at a distance $\rho_{x, y} \leq\left. 3\right|_{t}$. The propagating waves reach a typical depth $z$ of about half this distance $[35,36]$. If only a section of the light path crosses a region of interest (blue shaded circle), the effective path length inside this region needs to be considered only. Modeling the latter is difficult if the sample is accessed only from a single perspective. The problem can however be overcoming using optical tomography based on a numerical analysis of the signal obtained from a number of different source/detector pairs placed around the object [24].

\section{$7 \quad$ Laser Speckle Imaging}

Laser speckle imaging is a related approach that uses coherent laser light and measures the "absorption of correlation" [5, 27, 39-42] in the process of multiple scattering. It provides a measure for the local dynamic properties in a scattering medium. The underlying principle of LSI is equivalent to the one exploited in laser Doppler flowmetry, a method widely used in biomedical functional imaging of blood flow [5]. A moving object inside the tissue, such as a red blood cell, leads to a Doppler shift of the frequency of scattered light. Rather than analyzing Doppler frequency shifts LSI looks at temporal fluctuations of the scattered light in order to map dynamics. In LSI a digital camera records the surface intensity pattern of light reflected from the tissue. In the absence of movement (or for very short exposure time), there is no detectable fluctuation and the typical granular interference pattern, called speckle, has the same properties in the entire field of view. The spatially resolved speckle properties evolve in the presence of movement and thus can be a sensitive probe of blood flow dynamics. The strength and limitations of LSI are similar to the ones found in OIS. This is not surprising since both methods are founded on exactly the same physical principles (absorption and scattering of diffusely reflected light). The LSI measurement is rather easy to implement and the data acquisition is straightforward. Quantification, however, faces the same problems as OIS since knowledge about the exact path lengths is difficult to obtain from a single wide-field experiment. Moreover, the penetration depth is limited to the mm-range due to the wide-field 
illumination usually applied. Additional complications, specific to LSI and not present in OIS, are the limited signal to noise ratio and the sensitivity of the method to vibrations and body movements, for example, due to breathing and heartbeat [43].

\section{Diffuse Optical Tomography}

In contrast to the planar imaging methods discussed in the previous sections, diffuse optical tomography (DOT) attempts to reconstruct 3D images by acquiring multiple images from different perspectives or by using a set of point sources and detectors. The tomographic scheme consists in solving a number of equations for the unknown spatial distribution of scattering properties. It has been implemented for absorption, fluorescence, and speckledecorrelation $[3,5,24]$. Elaborate theoretical frameworks have been developed since the early 1990 [44]. Due to the additional information available, a numerical reconstruction is possible within some approximation (such as the diffusion equation for light propagation) without any prior knowledge about the medium. For small animals, NIR light is able to penetrate the whole body and thus diffuse transmission measurements can be performed for most possible combinations of source and detector position. Using fluorescence markers, the signal to noise ratio can be substantially improved. The existence of parameter-free theoretical model moreover allows quantification of the signal, for example, to extract the fluorophore density [24].

DOT type approaches have been applied to humans and neonates since the early 1990s [45]. Due to the limited penetration depth of NIR light transmission measurements are precluded and thus an optical topography approach is usually applied. It consists of placing an array of sources and detectors around the skull. With a spacing of typically $3 \mathrm{~cm}$ this provides a penetration depth of roughly $1.5 \mathrm{~cm}$. Based on tomographic reconstruction methods, a map of the absorption parameter can be obtained. Since blood is a strong absorber (Fig. 2), this approach allows mapping of the hemodynamic response in the brain cortex with $\mathrm{mm}$ resolution $[36,46]$.

\section{Summary and Conclusions}

The aim of this introductory chapter was to highlight some of the main physical and optical mechanism that make up the foundation of modern neuroimaging methods. Naturally, the limited space available only allows touching the very surface of this broad field. My modest goal was to remind the reader of some basic principle of image formation. I then focused on the important role of scattering in many modern imaging applications. As I have shown for the case 
of diffuse light general concepts exist that can be applied to a variety of imaging techniques. The complexity of applying these concepts to light propagation in a complicated heterogeneous medium such as living tissue, however, remains a challenging task until today.

\section{Acknowledgments}

Financial support from the Swiss National Science Foundation (Grants No. 126772 and 132736) and the Adolphe Merkle Foundation is gratefully acknowledged. I am grateful to Bruno Weber, Fritjof Helmchen, and Jorge Ripoll for valuable comments and discussions.

\section{References}

1. Pawley JB (2006) Handbook of biological confocal microscopy, 3rd edn. Springer, New York, NY

2. Murphy DB (2001) Fundamentals of light microscopy and electronic imaging. Wiley-Liss, New York

3. Yodh A, Chance B (1995) Spectroscopy and imaging with diffusing light. Phys Today 48(3): 34-40

4. Helmchen F, Denk W (2005) Deep tissue two-photon microscopy. Nat Methods 2(12): 932-940

5. Briers JD (2001) Laser Doppler, speckle and related techniques for blood perfusion mapping and imaging. Physiol Meas 22(4):R35-R66

6. Atkins PW, De Paula J (2002) Physical chemistry, 7th edn. W.H. Freeman, New York

7. Bastiaens PIH, Squire A (1999) Fluorescence lifetime imaging microscopy: spatial resolution of biochemical processes in the cell. Trends Cell Biol 9(2):48-52

8. Miyawaki A (2005) Innovations in the imaging of brain functions using fluorescent proteins. Neuron 48(2):189-199

9. Lakowicz JR (1999) Principles of fluorescence spectroscopy, 2nd edn. Kluwer, New York

10. Sakadzic S, Roussakis E, Yaseen MA, Mandeville ET, Srinivasan VJ, Arai K, Ruvinskaya S, Devor A, Lo EH, Vinogradov SA et al (2010) Two-photon high-resolution measurement of partial pressure of oxygen in cerebral vasculature and tissue. Nat Methods 7(9):755-759

11. Bohren CF, Huffman DR (1983) Absorption and scattering of light by small particles. Wiley, New York
12. Haiss F, Jolivet R, Wyss MT, Reichold J, BenBraham NB, Scheffold F, Krafft MP, Weber B (2009) Improved In vivo two-photon imaging after blood replacement by perfluorocarbon. J Physiol 587:3153-3158

13. Faber DJ, Aalders MCG, Mik EG, Hooper BA, van Gemert MJC, van Leeuwen TG (2004) Oxygen saturation-dependent absorption and scattering of blood. Phys Rev Lett 93(2):028102

14. Oheim M, Beaurepaire E, Chaigneau E, Mertz J, Charpak S (2001) Two-photon microscopy in brain tissue: parameters influencing the imaging depth. J Neurosci Methods 111(1):29-37

15. Huisken J, Swoger J, Del Bene F, Wittbrodt J, Stelzer EHK (2004) Optical sectioning deep inside live embryos by selective plane illumination microscopy. Science 305(5686):1007-1009

16. Mertz J (2011) Optical sectioning microscopy with planar or structured illumination. Nat Methods 8(10):811-819

17. Denk W, Strickler JH, Webb WW (1990) 2-Photon laser scanning fluorescence microscopy. Science 248(4951):73-76

18. Trachtenberg JT, Chen BE, Knott GW, Feng GP, Sanes JR, Welker E, Svoboda K (2002) Long-term in vivo imaging of experiencedependent synaptic plasticity in adult cortex. Nature 420(6917):788-794

19. Ripoll J (2012) Principles of diffuse light propagation: light propagation in tissues with applications in biology and medicine. World Scientific Publishing Company, Singapore

20. Crassous J, Erpelding M, Amon A (2008) Diffusive wave spectroscopy applied to the spatially resolved deformation of a solid. Phys Rev E Stat Nonlin Soft Matter Phys 78(4):046104 
21. Boffety M, Allain M, Sentenac A, Massonneau M, Carminati R (2008) Analysis of the depth resolution limit of luminescence diffuse optical imaging. Opt Lett 33(20):2290-2292

22. Azizi L, Zarychta K, Ettori D, Tinet E, Tualle JM (2009) Ultimate spatial resolution with diffuse optical tomography. Opt Express 17(14): 12132-12144

23. Deliolanis N, Lasser T, Hyde D, Soubret A, Ripoll J, Ntziachristos V (2007) Free-space fluorescence molecular tomography utilizing 360 degrees geometry projections. Opt Lett $32(4): 382-384$

24. Ntziachristos V, Ripoll J, Wang LHV, Weissleder R (2005) Looking and listening to light: the evolution of whole-body photonic imaging. Nat Biotechnol 23(3):313-320

25. Boas DA, Yodh AG (1997) Spatially varying dynamical properties of turbid media probed with diffusing temporal light correlation. J Opt Soc Am A 14(1):192-215

26. Boas DA, Campbell LE, Yodh AG (1995) Scattering and imaging with diffusing temporal field correlations. Phys Rev Lett 75(9): $1855-1858$

27. Briers JD (1996) Laser Doppler and timevarying speckle: a reconciliation. J Opt Soc Am A 13(2):345-350

28. Volker AC, Zakharov P, Weber B, Buck F, Scheffold F (2005) Laser speckle imaging with an active noise reduction scheme. Opt Express 13(24):9782-9787

29. Durduran T, Choe R, Baker WB, Yodh AG (2010) Diffuse optics for tissue monitoring and tomography. Rep Prog Phys 73(7):076701

30. Grinvald A, Lieke E, Frostig RD, Gilbert CD, Wiesel TN (1986) Functional architecture of cortex revealed by optical imaging of intrinsic signals. Nature 324(6095):361-364

31. Frostig RD, Lieke EE, Tso DY, Grinvald A (1990) Cortical functional architecture and local coupling between neuronal-activity and the microcirculation revealed by invivo high-resolution optical imaging of intrinsic signals. Proc Natl Acad Sci U S A 87(16): 6082-6086

32. Kohl M, Lindauer U, Royl G, Kuhl M, Gold L, Villringer A, Dirnagl U (2000) Physical model for the spectroscopic analysis of cortical intrinsic optical signals. Phys Med Biol 45(12): 3749-3764

33. Villringer A, Chance B (1997) Non-invasive optical spectroscopy and imaging of human brain function. Trends Neurosci 20(10):435-442

34. Joebsis FF (1977) Science 198 (4323): 1264-1267 DOI: 10.1126/science. 929199
35. Gibson AP, Hebden JC, Arridge SR (2005) Recent advances in diffuse optical imaging. Phys Med Biol 50(4):Rl-R43

36. Strangman G, Boas DA, Sutton JP (2002) Non-invasive neuroimaging using near-infrared light. Biol Psychiatry 52(7):679-693

37. Tian PF, Devor A, Sakadzic S, Dale AM, Boas DA (2011) Monte Carlo simulation of the spatial resolution and depth sensitivity of twodimensional optical imaging of the brain. J Biomed Opt 16(1):016006

38. Patterson MS, Chance B, Wilson BC (1989) Time resolved reflectance and transmittance for the noninvasive measurement of tissue opticalproperties. Appl Optics 28(12):2331-2336

39. Dunn AK, Bolay T, Moskowitz MA, Boas DA (2001) Dynamic imaging of cerebral blood flow using laser speckle. J Cereb Blood Flow Metab 21(3):195-201

40. Ayata C, Ozdemir Y, Dunn A, Atochin DN, Huang PL, Muzykantov VR, Murciano JC, Boas DA, Moskowitz MA (2003) Laser speckle-flowmetry: a novel two-dimensional technique for the study of cerebral blood flow in normal and ischemic mouse brain, in vivo. Stroke 34(1):251

41. Cheng HY, Luo QM, Zeng SQ, Chen SB, Cen J, Gong H (2003) Modified laser speckle imaging method with improved spatial resolution. J Biomed Opt 8(3):559-564

42. Weber B, Burger C, Wyss MT, von Schulthess GK, Scheffold F, Buck A (2004) Optical imaging of the spatiotemporal dynamics of cerebral blood flow and oxidative metabolism in the rat barrel cortex. Eur J Neurosci 20(10):2664-2670

43. Peuser J, Belhaj-Saif A, Hamadjida A, Schmidlin E, Gindrat AD, Volker AC, Zakharov P, Hoogewoud HM, Rouiller EM, Scheffold F (2011) Follow-up of cortical activity and structure after lesion with laser speckle imaging and magnetic resonance imaging in nonhuman primates. J Biomed Opt 16(9):096011

44. Arridge SR, Schweiger M, Hiraoka M, Delpy DT (1993) A finite-element approach for modeling photon transport in tissue. Med Phys 20(2):299-309

45. Gratton G, Corballis PM, Cho EH, Fabiani M, Hood DC (1995) Shades of gray-matternoninvasive optical-images of human brain responses during visual-stimulation. Psychophysiology 32(5):505-509

46. Koizumi H, Yamamoto T, Maki A, Yamashita Y, Sato H, Kawaguchi H, Ichikawa N (2003) Optical topography: practical problems and new applications. Appl Optics 42(16): 3054-3062 